\title{
STOCHASTIC TIME EVOLUTION OF ONE DIMENSIONAL INFINITE PARTICLE SYSTEMS
}

\author{
BY FRANK SPITZER ${ }^{1}$
}

1. Introduction. A familiar fact about Poisson systems of particles (random distribution) in Euclidean space $\mathbf{R}_{d}$ will serve as a suitable starting point for this survey. Let us denote such a Poisson system by $\mathscr{P}_{\rho}$, where $\rho>0$ is the constant particle density. $\mathscr{P}_{\rho}$ is then characterized by the number of particles $N_{B}$ in each bounded Borel set $B$ with volume $|B|$ being Poisson distributed with mean $\rho|B|$, and by the mutual independence of the occupation numbers $N_{B_{i}}$ corresponding to mutually disjoint $B_{i} \subset \mathbf{R}_{d}$. Consider now the following stochastic time evolution: each particle remains at rest for an exponential time with mean one, and then it jumps in such a way that its displacement is governed by a given probability measure $\nu$ on $\mathbf{R}_{d}$. All jumps and waiting times are assumed to be mutually independent random variables. Then, if the system is a Poisson system $\mathscr{P}_{\rho}$ at time $t=0$, it remains a Poisson system with the same density $\rho$ at all future $t>0$. We say that, for each $\rho>0$, $\rho_{\rho}$ is invariant under (is an equilibrium state for) the jump evolution with speed one and jump measure $\nu$. The simplicity of this state of affairs is due to the total lack of interaction between the motions of distinct particles. The same phenomenon occurs in continuous time: for example, let the particles perform independent Brownian motions, as in the discussion of Doob [2, pp. 404-407]. Again each $\mathscr{P}_{\rho}$ is an equilibrium state.

There is another stochastic time evolution which derives its simplicity from a similar lack of interaction between particles. We call it a birth and death evolution: each particle dies (disappears) with given death rate $\delta>0$, i.e. it lives for an exponential time with mean $\delta^{-1}$. However for each bounded Borel set $B \subset \mathbf{R}_{d}$ there is a constant rate $\beta|B|>0$ for the birth of a particle, which upon birth appears at some point uniformly distributed over $B$. Since the evolutions in disjoint sets $B_{1}$ and $B_{2}$ are independent, it is not difficult to construct the evolution as a Markov process whose state space is the set of all integer valued set functions (configurations) on $\mathbf{R}_{d}$. It is then trivial to verify that this evolution leaves the Poisson system $\mathscr{P}_{\rho}$ invariant if and only if $\rho=\beta / \delta$.

The above examples motivate current research efforts in this field. The aim is to study stochastic time evolutions with nontrivial interaction between particles, and in particular to obtain information about the ergodic behavior (equilibrium states, convergence to equilibrium) of such systems. The choice

An invited address delivered before the San Antonio, Texas meeting, January 23, 1976; received by the editors February 25, 1977.

AMS (MOS) subject classifications (1970). Primary 60J25, 60J75, 60J80, 60K 15, 60K35.

Key words and phrases. Infinite particle systems, stochastic time evolution, equilibrium states, time reversible equilibria.

${ }^{1}$ Research partially supported by the NSF at Cornell University. 
of natural evolutions to study has often come from statistical mechanics where the interaction is defined by a physically meaningful Hamiltonian based on a given potential $V$. In those cases when the time evolution has a time reversible equilibrium state, it then turns out that this equilibrium state must be a Gibbs state corresponding to the potential $V$. For stochastic time evolutions this remarkable fact was first established in the pioneering work of Kolmogorov [6]. He considered independent Brownian motions of a finite particle system on a compact manifold without boundary (say a torus). Each particle has a drift added to its standard Brownian motion, which depends on and constitutes its interaction with the other particles. Kolmogorov showed that there is a time reversible equilibrium state if and only if the drift vector for each particle is the gradient of its potential of interaction with the other particles. And, if so, then the time reversible equilibrium state is the Gibbs state corresponding to the interaction potential. This result has recently been extended from finite particle systems to infinite particle systems on $\mathbf{R}_{d}$ by $\mathbf{R}$. Lang [7].

Most other authors to date, in considering jump processes and birth and death (also called spin-flip) evolutions have found it expedient or necessary to replace $\mathbf{R}_{d}$ by a countable set such as the lattice space $\mathbf{Z}_{d}$. (See [8] for a recent survey and up to date bibliography.) Then the existence of the time evolution as a Markov process is easier to prove, especially when the interaction precludes multiple occupancy of sites of $\mathbf{Z}_{d}$. The state space is then the compact space $\{0,1\}^{\mathbf{Z}_{d}}$.

In the present study we shall restrict ourselves entirely to dimension $d=1$, in order to describe a class of stochastic evolutions whose interaction has no meaningful analogue in dimension higher than one. They will be called nearest particle evolutions and have the property that the speed of jumping, resp. the birth and death rates, depend only on the distances to the nearest particle on the left and on the right. In $\$ 2$ we shall describe a class of evolutions called jump evolutions with exclusion. They have the property that jumps which would interchange the order of particles on $\mathbf{R}$ are excluded. There we shall make plausible that the equilibrium states of such evolutions belong to the class of renewal processes, a natural generalization of the Poisson systems $\mathscr{P}_{\rho}$. The spacing density of the renewal process will be seen to be related in a natural way to the speed of the jump evolution with exclusion. The results will be in the form of conjectures, since the rigorous construction of these evolutions is an unsolved problem. In $\$ 3$ we shall discuss a similar generalization of birth and death evolutions with interaction, whose birth and death rates are allowed to depend on the distances to the nearest particles. Again the equilibrium state is a renewal process whose spacing density depends on the birth and death rates, and time reversibility will play a crucial role just as in the above mentioned work of Kolmogorov.

Just as in the case of $\$ 2$, the results of $\$ 3$ were purely formal at the time of this lecture (January 1976). Fortunately R. Holley and D. Stroock have succeeded since then in providing a satisfactory existence and uniqueness theory [5] for the evolutions in question, which enabled them to prove the conjectures in this part of the lecture. Therefore $\$ 3$ will be cast in the simpler framework of birth and death evolutions on $\mathbf{Z}$ instead of $\mathbf{R}$, for which $\mathbf{L}$. 
Gray [4] has obtained even stronger existence and uniqueness results than Holley and Stroock. It will be shown that the characterization of the (unique) time reversible equilibrium state hinges on a new characterization of renewal processes, which may be of independent interest. This characterization (which served as a model for Holley and Stroock's similar but deeper result on $\mathbf{R}$ in [5]) will be discussed in $\$ 4$.

Many of the ideas in $\S \S 3$ and 4 were developed jointly with Kenneth Logan, who used them in the related context of characterizing the time reversible equilibria for stochastic nearest neighbor time evolution on $\mathbf{Z}_{d}$ [9] (nearest neighbor-as opposed to nearest particle-means that the rates at a site in $\mathbf{Z}_{d}$ depend only on which neighboring sites are occupied).

2. Jump evolutions. First a formal definition of renewal processes, since our goal is to find jump evolutions whose equilibrium states are renewal processes.

Definition 2.1. For each strictly positive probability density $f$ on $\mathbf{R}^{+}$with finite expectation $\int_{0}^{\infty} x f(x) d x=m=\rho^{-1}$ ( $f$ is called the density of spacing) we define a point process on $\mathbf{R}$ as follows: place one particle at $-L$ and one at $R$ according to the joint density

(2.1) $\operatorname{Prob}[-L \in d x, R \in d y]=\rho f(x+y) d x d y, \quad x>0, y>0$.

Then place infinitely many particles to the left of $-L$ and to the right of $R$, with independent spacings given by the density $f$. This point process will be called $\Omega_{f}$ and the set of all renewal processes so obtained is $\stackrel{\leftrightarrow}{R}=\cup_{f} \mathrm{GR}_{f}$.

We shall say that $f \sim g$ or $\Omega_{f} \sim R_{g}$ if both $f$ and $g$ have positive means and $g(x)=c \exp (-\alpha x) f(x)$ for suitable $c>0$ and $\alpha \in \mathbf{R}$.

REMARK. The above construction due to Ryll-Nardzewski [10] defines a translation invariant point process. Note that $\varrho_{f}=\mathscr{P}_{\rho}$ when $f$ is an exponential density with mean $\rho^{-1}$, and that all the Poisson processes form one equivalence class.

Next we need a natural finite approximation of the infinite point process $\Re_{f}$ on $\mathbf{R}$ by an $N$-particle point process on a circle $T_{R}$ of radius $R$. We can think of $N$ distinguishable particles as a point $x=\left(x_{1}, \ldots, x_{N}\right) \in T_{R}^{N}$. Then the $N$ spacings between adjacent particles are well defined, up to cyclic permutation, as functions $\delta_{1}(x), \delta_{2}(x), \ldots, \delta_{N}(x)$, and we let

$$
\Phi_{R}^{(N)}(x)=c \prod_{k=1}^{N} f\left[\delta_{k}(x)\right], \quad x \in T_{R}^{N}
$$

where $c$ is the normalization such that $\Phi$ has integral one over $T_{R}^{N}$. It seems entirely natural that the point process determined by $\Phi_{R}^{(N)}$ should converge weakly to $h_{f}$ if $N \rightarrow \infty$ and $R \rightarrow \infty$ simultaneously, in such a way that the particle density $N /(2 \pi R)$ converges to $\rho=\left(\int x f(x) d x\right)^{-1}$. This is not hard to show [3]; in fact the same proof will show that when $N /(2 \pi R) \rightarrow \alpha>0$, then the point processes determined by $\Phi_{R}^{(N)}$ converge weakly to $\mathbb{R}_{g}$ where $g \sim f$ and $g$ has $\alpha=\left(\int x g(x) d x\right)^{-1}$, provided such a $g$ exists. This is so because $\Phi_{R}^{(N)}$ as defined by (2.2) depends not on $f$, but only on the equivalence class containing $f$.

Therefore we shall study time evolutions on $T_{R}^{N}$ whose behavior is inde- 
pendent of $R$, and if we find that their equilibrium state is $\Phi_{R}^{(N)}$, then it will be plausible to assume that the corresponding equivalence class of point processes $\Omega_{f}$ are equilibrium states of the corresponding time evolution of an infinite particle system on $\mathbf{R}$.

DEFINITION 2.2. A nearest particle jump evolution with speed change (either on $\mathbf{R}$ or $\left.T_{R}\right)$ is determined by a speed function $c(l, r)>0$ and a jump measure $\nu(d x)$, as follows: each particle jumps in time $d t$ with probability $c(l$, $r) d t$ when $l>0$ and $r>0$ are the distances to the nearest particle on the left and on the right. The displacement due to the jump is governed by the probability measure $\nu$ on $\mathbf{R}$.

THEOREM 2.3. Let $f$ be an admissible probability density in the sense of Definition 2.1 and let $\Phi=\Phi_{R}^{(N)}$ be as in (2.2). Then $\Phi$ is invariant under the evolution on $T_{R}$ in Definition 2.2, for any $\nu$, provided the speed function is of the form

$$
c(l, r)=f(l+r) / f(l) f(r), \quad l>0, r>0 .
$$

Proof. To simplify the notation, assume $\nu(d x)=\varphi(x) d x$ to be absolutely continuous. Then the semigroup of the evolution has the representation

$$
P_{t}(x, y)=\exp (t G)(x, y), x, y \in T_{R}^{N} \text {. }
$$

Now $\Phi$ will be an equilibrium state if

$$
\int_{T_{R}^{N}} \Phi(x) G(x, y) d x=0, \quad y \in T_{R}^{N},
$$

and a time reversible equilibrium if

$$
\Phi(x) G(x, y)=\Phi(y) G(y, x), \quad x, y \in T_{R}^{N} .
$$

We shall verify that (2.5) holds under the additional assumption that $\nu$ is symmetric, i.e. $\varphi(x)=\varphi(-x)$. Without this (2.5) does not hold but (2.4) is easy to verify, as in similar calculations in [11].

For each $\xi \in T_{R}$ let

$$
S_{k}(\xi) x=\left(x_{1}, x_{2}, \ldots, x_{k-1}, \xi, x_{k+1}, \ldots, x_{N}\right), \quad x \in T_{R}^{N} .
$$

Note that (2.5) holds if $x=y$. Also if $x \neq y$, then $G(x, y) \neq 0$ only if $y$ is of the form $S_{k}(\xi) x$, for some $1 \leqslant k \leqslant N$ and some $\xi \in T_{R}$, which means that the $k$ th particle jumps from $x_{k}$ to $\xi$. When the configuration is $x=$ $\left(x_{1}, \ldots, x_{N}\right)$, then, assuming (2.3), the speed of the $k$ th particle is

$$
\frac{f(l+r)}{f(l) f(r)}=\frac{\Phi_{R}^{(N-1)}\left(\pi_{k} x\right)}{\Phi_{R}^{(N)}(x)}
$$

where $\pi_{k} x=\left(x_{1}, x_{2}, \ldots, x_{k-1}, x_{k+1}, \ldots, x_{N}\right)$. Therefore (2.5) takes the form $\Phi_{R}^{(N)}(x) \frac{\Phi_{R}^{(N-1)}\left(\pi_{k} x\right)}{\Phi_{R}^{(N)}(x)} \varphi(\xi-x)=\Phi_{R}^{(N)}\left(S_{k}(\xi) x\right) \frac{\Phi_{R}^{(N-1)}\left(\pi_{k} S_{k}(\xi) x\right)}{\Phi_{R}^{(N)}\left(S_{k}(\xi) x\right)} \varphi(x-\xi)$, which is satisfied, since $\Phi_{R}^{(N-1)}\left(\pi_{k} S_{k}(\xi) x\right)=\Phi_{R}^{(N-1)}\left(\pi_{k} x\right)$.

This theorem then leads to the

CONJECTURE. The nearest particle jump evolution with speed change given by $c(l, r)=f(l+r) / f(l) f(r)$ has as equilibrium states all , $_{g}$ such that $g \sim f$. 
It has to be understood, however, that the very existence of these time evolutions is still in question in all cases except when $f$ is an exponential density. Once this is settled one could attempt to prove an even stronger conjecture, of a type which will be discussed in detail in \$3. It states that if an evolution of the type discussed has a time reversible equilibrium state, then this state must be a renewal process, or a convex combination of such objects-and that this can happen only if the speed is a constant multiple of the speed in (2.3).

We proceed to another class of jump processes with exclusion of jumps which would change the order of particles.

Definition 2.3. A nearest particle jump evolution with speed change and exclusion (either on $\mathbf{R}$ or $T_{R}$ ) is defined just as in Definition 2.2., except that jumps are excluded which would reverse order.

THEOREM 2.4. Let $f$ be an admissible probability density in the sense of Definition 2.1, and let $\Phi=\Phi_{R}^{(N)}$ be as in (2.2). Then $\Phi$ is invariant under the evolution on $T_{R}$ in Definition 2.3, for any $v$, provided the speed function is of the form

$$
c(l, r)=h(l+r) / f(l) f(r), \quad l>0, r>0,
$$

where $h$ is any strictly positive measurable function.

We omit the proof since it is similar to that of Theorem 2.3 and was already given, for the case of $h \equiv 1$, in [11, pp. 261-263]. Also the resulting conjectures are analogous to those following Theorem 2.3 and were discussed in [11, pp. 284-289]. The existence of the time evolution on $\mathbf{R}$ remains an open problem.

3. Birth and death evolutions. Here the plan is to build an interaction into birth and death evolutions by making the birth and death rates depend on the distances $l$ and $r$ to the nearest particles on the left and on the right. Since R. Holley and D. Stroock have recently [5] developed this theory for infinite systems on $\mathbf{R}$, we shall treat the technically simpler case of $\mathbf{Z}$.

An admissible spacing density will be a probability density $f$ with

$$
\sum_{k=1}^{\infty} f(k)=1, \quad \sum_{k=1}^{\infty} k f(k)=m=\rho^{-1}<\infty, f(k)>0 \text { for } k=1,2,3, \ldots
$$

The translation invariant renewal process on $\mathbf{Z}$ corresponding to such a sequence $f$ will be denoted $\Re_{f}$ as in $\$ 2$ on $\mathbf{R}$. It is the probability measure $\mu$ on $\Omega=\{0,1\}^{\mathbf{Z}}$ which is uniquely determined by the cylinder set probabilities

$$
\mu\left[A_{k_{1} k_{2}, \ldots, k_{j}}^{k}\right]=m^{-1} f\left(k_{1}\right) f\left(k_{2}\right) \cdots f\left(k_{j}\right)
$$

where $A_{k_{1} k_{2}}^{k} \cdots k_{j}$ denotes the event that $\omega_{k}=\omega_{k+k_{1}}=\cdots=\omega_{k+k_{1}+k_{2}+\cdots+k_{j}}$ $=1$ and $\omega_{i}=0$ for all other integers $i$ between $k$ and $k+k_{1}+k_{2}+\cdots+$ $k_{j}$. Finally $\stackrel{\leftrightarrow}{R}$ denotes the union of ${ }^{\prime} R_{f}$ over all admissible $f$.

A nearest particle birth and death evolution has been shown to exist by $L$. Gray ([14], Theorem 4.4) in the following sense. Let $\Omega^{\prime} \subset \Omega$ be the set of configurations $\omega=\left\{\omega_{k} ; k \in \mathbf{Z}\right\} \in \Omega$ such that $\omega_{k}=1$ for infinitely many positive and infinitely many negative values of $k$. Let $\beta(l, r)$ and $\delta(l, r)$ be strictly positive functions on $l \geqslant 1, r \geqslant 1$ and such that $\delta$ is bounded. (The boundedness of $\delta$ 
is a natural condition because it prevents the particle system from dying out in a finite time.) Let

$$
c_{k}(\omega)= \begin{cases}\beta(l, r) & \text { if } \omega_{k}=0, \\ \delta(l, r) & \text { if } \omega_{k}=1,\end{cases}
$$

whenever $\omega_{k-1}=\omega_{k+r}=1$ and $\omega_{j}=0$ for $k-l<j<k+r$, and $j \neq k$. For each $k \in \mathbf{Z}$ and each $\omega \in \Omega^{\prime}$ let $\omega^{k} \in \Omega^{\prime}$ be the configuration which agrees with $\omega$ at all sites $j \neq k$ while $\left(\omega^{k}\right)_{k}=1-\omega_{k}$. Finally let $\mathscr{D}\left(\Omega^{\prime}\right)$ be the set of right continuous functions with left hand limits from $\mathbf{R}^{+}$into $\Omega^{\prime}$. Then there exists one and only one (strong) Markov process $\omega_{t}, t \geqslant 0$, with state space $\Omega^{\prime}$, whose trajectories lie in $\mathscr{D}\left(\Omega^{\prime}\right)$ and which is such that the domain of its pointwise generator $G$ contains the cylinder functions and is given by

$$
G h(\omega)=\sum_{k \in \mathbf{Z}} c_{k}(\omega)\left[h\left(\omega^{k}\right)-h(\omega)\right], \quad \omega \in \Omega^{\prime},
$$

for every cylinder function (depending only on finitely many coordinates) $h$.

A probability measure $\mu$ on $\Omega^{\prime}$ will be called an equilibrium state for the above time evolution (Markov process) $\omega(t)$ if

$$
\left.E_{\mu} f[\omega(t)]=E_{\mu} f \omega(0)\right], \quad t \geqslant 0,
$$

for every cylinder function $f$. It will be called a time reversible equilibrium state if it is an equilibrium state and in addition the stationary process $\omega(t)$, $t \in \mathbf{R}$, which is in state $\mu$ for all $t \in \mathbf{R}$ is invariant under the reflection $t \rightarrow-t$. It is known ([4], Theorem 3.21 and Theorem 4.4) that this happens if and only if

$$
\left.E_{\mu} f \omega(t)\right] g[\omega(0)]=E_{\mu} f[\omega(0)] g[\omega(t)], \quad t \geqslant 0,
$$

for all pairs of cylinder functions $f$ and $g$ and that this in turn is equivalent to

$$
\int \mu(d \omega)[f(\omega) G g(\omega)-g(\omega) G f(\omega)]=0
$$

for all pairs of cylinder functions $f$ and $g$.

The above results provide the appropriate setting for

THEOREM 3.1. (a) Suppose that a nearest particle birth and death evolution has rates $\beta(l, r)>0$ and $\delta(l, r)>0$ with $\delta(l, r)$ bounded, which satisfy the equation

$$
\frac{\beta(l, r)}{\delta(l, r)}=\frac{f(l) f(r)}{f(l+r)}, \quad l \geqslant 1, r \geqslant 1,
$$

where $f$ is an admissible spacing density. Then the renewal process $\mu=\Omega_{f}$ is a time reversible equilibrium state.

(b) Suppose, conversely, that we have a nearest particle birth and death evolution with $\beta(l, r)>0, \delta(l, r)>0$ and $\delta(l, r)$ bounded, and such that it possesses a time reversible equilibrium state $\mu$. Then it follows that $\mu=\mathrm{R}_{f}$ for some admissible spacing density $f$, and furthermore the rates must satisfy equation (3.5).

We illustrate the theorem by a concrete

EXAMPLE. Let $\delta(l, r) \equiv 1$ and $\beta(l, r)=A(1 / l+1 / r)^{2}, l \geqslant 1, r \geqslant 1$, where $A>0$ is a given parameter. Then the evolution exists in the sense described. By Theorem 3.1 it has a time reversible equilibrium state $\mu$ if and only if there 
is an admissible spacing density $f$ such that

$$
\frac{f(l) f(r)}{f(l+r)}=A\left(\frac{1}{l}+\frac{1}{r}\right)^{2}, \quad l \geqslant 1, r \geqslant 1,
$$

and then $\mu=\Re_{f}$. To find all possible $f$ let $g(k)=k^{2} f(k), k \geqslant 1$. Then $g(l) g(r)=A g(l+r)$ for $l \geqslant 1, r \geqslant 1$, so that $g(k)=A e^{-\alpha k}$ for some real $\alpha$ or $f(k)=A k^{-2} e^{-\alpha k}$. But for $f$ to be admissible we must have

$$
A \sum_{1}^{\infty} k^{-2} e^{-\alpha k}=1, \quad A \sum_{1}^{\infty} k^{-1} e^{-\alpha k}<\infty,
$$

which works (for some $\alpha>0$ ) if and only if

$$
A>\left(\sum_{1}^{\infty} k^{-2}\right)^{-1}=\frac{6}{\pi^{2}} \text {. }
$$

Hence we have to distinguish two cases

(i) $A>6 / \pi^{2}$. By Theorem 3.1 there exists a unique time reversible equilibrium state $\mu=\Omega_{f}$, with

$$
f(k)=A k^{-2} e^{-\alpha k}, \quad k \geqslant 1,
$$

where $\alpha$ is the unique solution of $A \Sigma_{1}^{\infty} k^{-2} e^{-\alpha k}=1$.

(ii) $A \leqslant 6 / \pi^{2}$. By Theorem 3.1 there is no time reversible equilibrium state.

There is an obvious conjecture for what "goes wrong" in case (ii). It is that the birth rates are then sufficiently small compared to the death rates so that the particle system "dies out" in the weak sense that the probability that $(\omega(t))_{k}=0$ tends to one as $t \rightarrow \infty$ for each $k \in Z$, for any initial configuration in $\Omega^{\prime}$. This has indeed been proved by $R$. Holley (oral communication) by coupling methods, based on comparison of the process to one on a large finite interval with both end points occupied. This implies that when $A \leqslant 6 / \pi^{2}$ there is not even an equilibrium state $\mu$ such that $\mu\left(\Omega^{\prime}\right)=1$. When $A=6 / \pi^{2}$ however, it is not known whether there are equilibrium states which are not time reversible.

Proof of Theorem 3.1. Equation (3.4) is the necessary and sufficient condition for $\mu$ to be a time reversible equilibrium state. In view of (3.3) it takes the form

$$
\int \sum_{k \in \mathbf{Z}} c_{k}(\omega)\left[h(\omega) g\left(\omega^{k}\right)-h\left(\omega^{k}\right) g(\omega)\right] \mu(d \omega)=0,
$$

for pairs of cylinder functions $g$ and $h$. It can be simplified by

LEMMA 3.2. Equation (3.6) holds if and only if

$$
\int c_{0}(\omega) \varphi(\omega) \mu(d \omega)=\int c_{0}(\omega) \varphi\left(\omega^{0}\right) \mu(d \omega)
$$

for all cylinder functions $\varphi$.

To prove the lemma assume (3.7). In view of the translation invariance of the rates $c_{k}(\cdot)$ it implies

$$
\int c_{k}(\omega) \varphi(\omega) \mu(d \omega)=\int c_{k}(\omega) \varphi\left(\omega^{k}\right) \mu(d \omega), \quad k \in \mathbf{Z} .
$$

Letting $\varphi(\omega)=h(\omega) g\left(\omega^{k}\right)$, we see that (3.6) holds. Conversely suppose (3.6) holds and choose for some finite $A \subset \mathbf{Z}, j \in A$, 


$$
h(\omega)=\prod_{k \in A} \omega_{k}, \quad g(\omega)=h\left(\omega^{j}\right) .
$$

Then $h(\omega) g\left(\omega^{k}\right)-h\left(\omega^{k}\right) g(\omega)=0$ unless $k=j$. Therefore (3.6) implies that (3.7) holds for $\varphi=h$. By linearity (3.7) must hold for all cylinder functions $\varphi$.

To prove part (a) of Theorem 3.1 we now assume (3.5) and have to verify (3.7) when $\mu=\Omega_{f}$. We may in fact assume that the function $\varphi$ in (3.7) is the indicator of an event of the form $A_{l r} \cap B$ where

(3.8) $A_{l r}=\left[\omega: \omega_{-l}=\omega_{r}=\omega_{0}=1 ; \omega_{k}=0\right.$ for all $\left.-l<k<r, k \neq 0\right]$

and $B$ is an event specifying a finite number of coordinates $\omega_{j}$ with $j$ outside the interval $[-l, r]$. Note that $c_{0}(\omega)=\beta(l, r)$ on the set where $\varphi\left(\omega^{0}\right)=1$ and $\delta(l, r)$ on the set of $\omega$ such that $\varphi(\omega)=1$. Therefore (3.7) reduces to

$$
\frac{\beta(l, r)}{\delta(l, r)}=\frac{\int \varphi(\omega) \mu(d \omega)}{\int \varphi\left(\omega^{0}\right) \mu(d \omega)} .
$$

In view of the definition of $\varphi$, and using the fact that $\mu=\Omega_{f}$, we get, letting $\tilde{A}_{l r}=\left[\omega: \omega^{0} \in A_{l r}\right]$,

$$
\mu\left(A_{l r}\right)=f(l) f(r) / m, \quad \mu\left(\tilde{A}_{l r}\right)=f(l+r) / m
$$

and

$$
\frac{\int \varphi(\omega) \mu(d \omega)}{\int \varphi\left(\omega^{0}\right) \mu(d \omega)}=\frac{\mu\left(A_{l r} \cap B\right)}{\mu\left(\tilde{A}_{l r} \cap B\right)}=\frac{\mu\left(A_{l r}\right)}{\mu\left(\tilde{A}_{l r}\right)}=\frac{f(l) f(r)}{f(l+r)},
$$

which together with (3.9) complete the proof of part (a) of Theorem 3.1.

To prove part (b) of Theorem 3.1 one proceeds in a quite similar fashion, up to a point. We assume that $\mu$ is a time reversible equilibrium state for the time evolution and conclude just as above that it must satisfy the identity

$$
\frac{\mu\left(A_{l r} \cap B\right)}{\mu\left(\tilde{A}_{l r} \cap B\right)}=\frac{\beta(l, r)}{\delta(l, r)} .
$$

The proof will then be complete if we can deduce from (3.9) that $\mu$ must be a renewal process $\Omega_{f}$ for some admissible spacing density $f$. This will be done in the next section, in a theorem which translates the information we have about $\mu$ at this point into the statement that $\mu=\left(R_{f}\right.$ for some $f$. Let us summarize this information.

(i) $\mu(A)>0$ for finite cylinder sets $A \subset \Omega$.

This is clear since the birth and death rates are positive.

(ii) $\mu\left(\Omega^{\prime}\right)=1$, i.e. $\mu\left[\omega_{k}=1\right.$ i.o. for $k>0$ and also for $\left.k<0\right]=1$ which follows from Gray's existence theorem for the time evolution.

(iii) The conditional probability that $\omega_{n}=1$, given $\omega$ for all $k \neq n$ depends only on the distances $l$ and $r$ from the site $n$ to the nearest occupied sites on the left and on the right of $n$.

This most crucial property (iii) is a consequence of (3.10), since the conditional probability in question may be written

$$
\frac{\mu\left(A_{l r} \cap B\right)}{\mu\left(A_{l r} \cap B\right)+\mu\left(\tilde{A}_{l, r} \cap B\right)}=\frac{\beta(l, r)}{\delta(l, r)+\beta(l, r)},
$$


which evidently is independent of the event $B$ and depends only on the spacings $l$ and $r$.

4. A characterization of renewal processes. Let $i R=U \Omega_{f}$ be the class of renewal processes described in the beginning of $\$ 3$, and let $\xi$ denote the class of processes satisfying properties (i), (ii) and (iii) at the end of the last section. We shall show that $\mathcal{G}=R$ and since the inclusion $R \subset \mathcal{G}$ is quite obvious, we begin immediately by assuming that $\mu \in \mathcal{G}$ and we shall show that $\mu \in \mathbb{R}$. The proof is divided into eight steps.

Step 1. Choose the events $A_{l r}$ as in (3.7) and $\tilde{A}_{l r}=\left[\omega: \omega^{0} \in A_{l r}\right]$, and call

$$
\mu\left[A_{l r}\right] / \mu\left[\tilde{A}_{l r}\right]=g(l, r) .
$$

Then there is a strictly positive sequence $a_{k}$, such that

$$
g(l, r)=a_{l} a_{r} / a_{l+r} .
$$

Proof. By using property (iii) one can express the ratio

$$
\frac{\mu\left[\omega_{0}=\omega_{j}=\omega_{j+k}=\omega_{j+k+n}=1, \omega_{i}=0 \text { for all other } 0<i<j+k+n\right]}{\mu\left[\omega_{0}=\omega_{j+k+n}=1, \omega_{i}=0 \text { for all } 0<i<j+k+n\right]}
$$

in two different ways in terms of the function $g$. This yields the functional equation

$$
g(j, k) g(j+k, n)=g(k, n) g(j, k+n) .
$$

To solve this equation let $t_{n}=\prod_{i=1}^{n} g(i, 1)$, define $a_{k}=t_{k}^{-1} g(k, 1)$ and verify that 4.2 holds.

Step 2. Let $A_{k_{1} k_{2} \cdots k_{j}}^{k}$ be the basic cylinder events used in equation (3.1). Let $n=k_{1}+k_{2}+\cdots+k_{j}$. Then there exists a family of constants $c_{n}^{(k)}$, with $n \geqslant 0, k \in \mathbf{Z}$, such that

$$
\mu\left(A_{k_{1} k_{2}}^{k} \cdots k_{j}\right)=c_{n}^{(k)} a_{k_{1}} a_{k_{2}} \cdots a_{k_{j}} .
$$

PRoof. First define $c_{0}^{(k)}=\mu\left[\omega_{k}=1\right]$. Then define $c_{n}^{(k)}$ by setting $\mu\left(A_{n}^{k}\right)=$ $c_{n}^{(k)} a_{n}$ for $n \geqslant 1$. The rest follows by induction on $j$ from Step 1. For example, if $k_{1}+k_{2}=n$, then by property (iii) and Step 1 we have

$$
\mu\left(A_{k_{1} k_{2}}^{k}\right)=\mu\left(A_{n}^{k}\right) g\left(k_{1}, k_{2}\right)=\frac{c_{n}^{(k)} a_{n} \cdot a_{k_{1}} a_{k_{2}}}{a_{n}}=c_{n}^{(k)} a_{k_{1}} a_{k_{2}} \text {. }
$$

Step 3. For $n \geqslant 0$

$$
c_{n}^{(k)}=\sum_{j=1}^{\infty} c_{n+j}^{(k)} a_{j}, \quad k \in \mathbf{Z} .
$$

Proof. By property (ii) of $\mu$ we know that $\omega_{i}=1$ for some $i>k$ with probability one. If $j+k+n$ is the first index after $k$ where this occurs, we obtain from Step 2 that $\mu\left(A_{n}^{k}\right)$ can be decomposed as follows:

$$
c_{n}^{(k)} a_{n}=\sum_{j=1}^{\infty} c_{n+j}^{(k)} a_{n} a_{j}
$$

Step 4. If $\left\{a_{k}\right\}_{k \geqslant 1}$ is a given strictly positive sequence such that the equation 


$$
c_{n}=\sum_{j=1}^{\infty} c_{n+j} a_{j}, \quad n \geqslant 0,
$$

has a nonnegative solution $c_{n}, n \geqslant 0$ (not identically zero), then the sequence $\left\{a_{k}\right\}$ must be such that the equation

$$
\sum_{k=1}^{\infty} a_{k} t^{k}=1
$$

has a unique positive root $t_{0}$. The solution $\left\{c_{n}\right\}$ must be of the form $c_{n}=\alpha t_{0}^{n}$, $n \geqslant 0$, for some $\alpha>0$.

Proof. We shall use standard Martin boundary theory. Without loss of generality we may assume $c_{0}=1$, since (4.5) shows that $c_{0}>0$. Let $S$ be the convex set of excessive sequences, i.e.,

$$
S=\left\{c: c_{0}=1, c_{n} \geqslant 0 \text { and } c_{n} \geqslant \sum_{j=1}^{\infty} c_{n+j} a_{j} \text { for } n \geqslant 0\right\} .
$$

The set $S$ is compact in the topology of pointwise convergence since $c_{j} \leqslant a_{j}^{-1}$ for each $j \geqslant 1$ and because the limit of excessive functions is again excessive. $S$ is also metrizable. By an integral representation theorem of Choquet, [1, Theorem 27.6], the sequence $c$ admits an integral representation in terms of the extreme points of $S$. Each of the extreme points occurring in this representation must satisfy (4.5) (be harmonic) since $c$ does. Therefore we shall try to find the harmonic extreme points of $S$. Suppose that $c$ is one of them. Then

$$
c_{n}=\sum_{j=1}^{\infty} a_{j} c_{j} \frac{c_{n+j}}{c_{j}}=\sum_{j=1}^{\infty} a_{j} c_{j} \varphi_{j}(n),
$$

where $\sum_{1}^{\infty} a_{j} c_{j}=1$ and each $\varphi_{j}$ is harmonic. Since $c$ is extreme we have $\varphi_{j}(n)=c_{n}$ for $n \geqslant 1$ for any value of $j \geqslant 1$. It follows that

Consequently

$$
c_{n+j}=c_{j} c_{n}, \quad n \geqslant 1, j \geqslant 1 .
$$

$$
c_{n}=\alpha t^{n} \quad \text { for some } t>0 .
$$

Substituting into (4.5) it follows that $t$ is the (obviously unique) positive solution of $\Sigma a_{k} t^{k}=1$. Thus $S$ has only one harmonic extremal. Therefore it is the desired solution of (4.5) and Step 4 is proved.

Step 5 . The constants $c_{n}^{(k)}$ in Step 2 satisfy

$$
c_{n}^{(k)}=\alpha_{k} t^{n}, \quad n \geqslant 0, k \in \mathbf{Z},
$$

where $t$ is the unique positive root of $\Sigma_{1}^{\infty} a_{k} t^{k}=1$, and $\alpha_{k}, k \in \mathbf{Z}$, are constants.

Proof. Immediate by applying the result in Step 4 to the equations (4.4).

Step 6. Define the sequence $f(k), k \geqslant 1$, by $f(k)=a_{k} t^{k}$, with $t$ defined in Step 5. Then

$$
\mu\left(A_{k_{1} k_{2} \cdots k_{j}}^{k}\right)=\alpha_{k} f\left(k_{1}\right) f\left(k_{2}\right) \cdots f\left(k_{j}\right)
$$

where $f(k)>0$ and $\Sigma_{1}^{\infty} f(k)=1$. 
Proof. Immediate from Steps 2 and 5.

Step 7. $\alpha_{k}=\alpha>0$ for all $k \in \mathbf{Z}$.

Proof. From Step 6 we have $\mu\left[\omega_{k}=\omega_{k+1}=1\right]=\alpha_{k} f(1)$, where the $\alpha_{k}$ are unknown positive constants (positive by property (i) of $\mu$ ). But all our inductions could have been performed moving to the left instead of to the right on Z. This would have yielded $\mu\left[\omega_{k+1}=\omega_{k}=1\right]=\beta_{k+1} f(1)$, where $\left\{\beta_{k}\right\}$ is another sequence of unknown positive constants. Similarly we get

$$
\mu\left[\omega_{k}=\omega_{k+n}=1\right]=\alpha_{k} f(n)=\beta_{k+n} f(n), \quad k \in \mathbf{Z}, n \geqslant 1 .
$$

Since $f(n)>0$ we get $\alpha_{k}=\beta_{k+n}$ for all $k \in \mathbf{Z}, n \geqslant 1$ which gives $\alpha_{k} \equiv \beta_{k} \equiv$ $\alpha>0, k \in \mathbf{Z}$.

Step 8 . The sequence $f(n), n \geqslant 1$, has finite mean, in fact for $\alpha$ as in Step 7

$$
\sum_{k=1}^{\infty} k f(k)=\frac{1}{\alpha}<\infty
$$

Proof. By Steps 6 and 7 and property (ii) of $\mu$

$$
\begin{aligned}
1-\alpha & =\mu\left[\omega_{0}=0\right]=\sum_{k=1}^{\infty} \sum_{l=1}^{\infty} \mu\left[\omega_{-k}=\omega_{l}=1, \omega_{j}=0 \text { for }-k<j<l\right] \\
& =\sum_{k=1}^{\infty} \sum_{l=1}^{\infty} \alpha f(k+l)=\alpha \sum_{j=1}^{\infty}(j-1) f(j)=\alpha\left[\sum_{j=1}^{\infty} j f(j)-1\right],
\end{aligned}
$$

which gives the desired result.

By Steps 6 and 8 the sequence $\{f(k)\}$ which we have defined is an admissible spacing density for a renewal process $\Omega_{f}$. By Steps 6 and 7 it follows that $\mu$ has the same cylinder set probabilities which in (3.1) were used to characterize $\Re_{f}$. Therefore $\mu=\Re_{f}$ which completes the proof.

\section{BIBLIOGRAPHY}

1. G. Choquet, Lectures on analysis. Vol. II, Benjamin, New York, 1969. MR 40 \#3253.

2. J. L. Doob, Stochastic processes, Wiley, 1953.

3. M. Fisher and B. Widom, Decay of correlations in linear systems, J. Chem. Phys. 9 (1969), 3756-3772.

4. L. F. Gray, Controlled spin-flip systems, Ph. D. Dissertation, Cornell University, 1977 (to appear).

5. R. Holley and D. W. Stroock, Nearest neighbor birth and death processes on the real line, Acta Math. (to appear).

6. A. N. Kolmogorov, Zur statistischen Umkehrbarkeit der Naturgesetze, Math. Ann. 113 (1937), 766-772.

7. R. Lang, Unendlich dimensionale Wiener Prozesse mit Wechselwirkung. I, Z. Wahrscheinlichkeitstheorie und Verw. Gebiete 38 (1977), 55-72.

8. T. M. Liggett, The stochastic evolution of infinite systems of interacting particles, Lecture Notes in Math., Springer-Verlag, Berlin and New York (to appear).

9. K. G. Logan, Time reversible evolutions in statistical mechanics, Ph. D. Dissertation, Cornell University, 1974.

10. C. Ryll-Nardzewski, Remarks on processes of calls, Proc. Fourth Berkeley Sympos. Math. Statist and Prob., Vol. II, Univ. of California Press, Berkeley, Calif., 1961, pp. 455-465. MR 25 \#3575.

11. F. Spitzer, Interaction of Markov processes, Advances in Math. 5 (1970), 246-290.

Department of Mathematics, Cornell University, Ithaca, New York 14853 